\title{
Atividade antimicrobiana de Senecio heterotrichius DC. (Asteraceae)
}

\author{
Leandro Nicolodi Francescato', Regis Augusto Norbert Deuschle1, Carlos Augusto Mallmann², \\ Sydney Hartz Alves ${ }^{1,3}$, Berta Maria Heinzmann ${ }^{1 *}$ \\ 'Departamento de Farmácia Industrial, Centro de Ciências da Saúde, Universidade Federal de Santa Maria, \\ ${ }^{2}$ Departamento de Medicina Veterinária Preventiva, Universidade Federal de Santa Maria, ${ }^{3}$ Departamento de \\ Microbiologia, Universidade Federal de Santa Maria
}

*Correspondência:

B. M. Heinzmann

Departamento de Farmácia Industrial Centro de Ciências da Saúde

Universidade Federal de Santa Maria Prédio 26, Campus Universitário 97105-900 - Santa Maria - RS, Brasil

E-mail: berta.heinzmann@gmail.com
Neste trabalho é relatada a avaliação da atividade antimicrobiana dos extratos diclorometânico e etanólico, obtidos por maceração das partes aéreas de $\mathrm{S}$. heterotrichius pelo método de microdiluição em caldo, frente a patógenos bacterianos e fúngicos. O extrato diclorometânico evidenciou boa atividade inibitória frente a Candida krusei (CIM de 0,25 mg/mL) e moderada atividade frente a Staphylococcus aureus (CIM de 2,5 $\mathrm{mg} / \mathrm{mL}$ ). O extrato etanólico mostrou-se inativo frente aos microrganismos testados. Também foi isolado o constituinte majoritário do extrato diclorometânico, cuja análise espectroscópica indicou tratar-se de um sesquiterpeno, identificado como germacreno $D$.

\section{INTRODUÇÃO}

O gênero Senecio (tribo Senecioneae, família Asteraceae) constitui um grupo de plantas cosmopolitas, não muito homogêneo (Jeffrey et al., 1977), presente em regiões frias e tropicais, principalmente na Europa, África, Américas Central e do Sul. Das mais de 2000 espécies amplamente distribuídas pela superfície terrestre, cerca de 85 crescem no Brasil (Cabrera, Klein, 1975).

Embora espécies de Senecio apresentem hepatotoxicidade reconhecida devido à presença de alcalóides pirrolizidínicos (Smith, Culvenor, 1981), várias delas são utilizadas na medicina popular de diferentes países, sendo em alguns casos, empregadas no tratamento de infecções (Kelmanson, Jäger, Van Staden, 2000; Portillo et al., 2001; Navarro García et al., 2003).

A utilização de plantas pertencentes ao gênero Senecio como medicinais pode ser explicada pela presença de diferentes classes de metabólitos secundários, uma vez que são ricas em monoterpenóides (Pérez, Agnese,
Cabrera, 1999), sesquiterpenóides (Dupré et al., 1991; Bohlmann, Ziesche, 1981), triterpenóides (Abdo et al., 1992) e flavonóides (Dupré et al., 1991; Mansour, Saleh, 1981), entre outros. As atividades biológicas já comprovadas para estas espécies são as mais variadas, como, por exemplo, propriedades inseticidas (Pascual-Villalobos, Robledo, 1998), atividades antimicrobiana (Cos et al., 2002; Navarro García et al., 2003; Loizzo et al., 2006), citotóxica (Loizzo et al., 2006), antiviral (Fortin et al., 2002) e antioxidante (Liu, Ng, 2000).

Senecio heterotrichius DC., popularmente conhecido como "catião-melado", cresce como um arbusto no sul do Brasil, Uruguai e nordeste da Argentina. Floresce nos meses de outubro e novembro (Cabrera, Klein, 1975) e comporta-se como invasora de culturas e pastagens nativas.

O presente trabalho descreve a avaliação da atividade antimicrobiana dos extratos diclorometânico e etanólico de $S$. heterotrichius, bem como o isolamento de um dos principais constituintes do primeiro. 


\section{MATERIAL E MÉTODOS}

\section{Material vegetal}

Partes aéreas frescas de Senecio heterotrichius DC. foram coletadas em abril de 2002 no município de Porto Alegre, RS, Brasil, e identificadas no Laboratório de Botânica da Faculdade de Farmácia da UFRGS. Material testemunha encontra-se depositado no Herbário do Departamento de Biologia da UFSM sob o número SMDB 8935.

\section{Obtenção dos extratos}

As partes aéreas frescas $(1193,5 \mathrm{~g})$ foram divididas e extraídas por maceração, sucessivamente com diclorometano e etanol, duas vezes cada, por 8 dias. Após a concentração dos macerados sob pressão reduzida, foram obtidos os extratos diclorometânico $\left(\mathrm{CH}_{2} \mathrm{Cl}_{2}\right)$ e etanólico (EtOH) com rendimento de $12,054 \mathrm{~g}(1,01 \%)$ e $43,3 \mathrm{~g}$ $(3,63 \%)$, respectivamente.

\section{Atividade antimicrobiana}

A avaliação da atividade antimicrobiana dos extratos $\mathrm{CH}_{2} \mathrm{Cl}_{2}$ e EtOH foi realizada através do método de microdiluição em caldo, previamente descrito por Deuschle et al. (2006), com base nos documentos M27-A2 (NCCLS, 2002) para fungos leveduriformes e M100-S12 (NCCLS, 2002) para bactérias.

Previamente aos testes, os cultivos bacterianos foram ativados através de subcultivos em ágar Mueller-Hinton durante 24 h a $35^{\circ} \mathrm{C}$, enquanto que os fungos foram subcultivados em ágar Sabouraud dextrose, $30-35^{\circ} \mathrm{C} / 48 \mathrm{~h}$. Após a ativação, padronizou-se o inóculo, que consistiu na preparação de uma suspensão bacteriana em solução fisiológica esterilizada a $0,9 \%$, com turvação similar ao tubo 0,5 da Escala Mac Farland ( $\left.1 \times 10^{8} \mathrm{UFC} / \mathrm{mL}\right)$. A seguir, esta suspensão foi diluída a 1:10 (1 x $\left.10^{7} \mathrm{UFC} / \mathrm{mL}\right) \mathrm{em}$ solução fisiológica esterilizada a $0,9 \%$, e volumes de $10 \mu \mathrm{L}$ foram então transferidos para as cavidades de microplaca esterilizada, contendo $200 \mu \mathrm{L}$ de caldo Mueller-Hinton acrescido das diferentes concentrações finais do extrato a ser testado, resultando num inóculo final de aproximadamente $5 \times 10^{5} \mathrm{UFC} / \mathrm{mL}$. As placas com patógenos bacterianos foram incubadas a $37^{\circ} \mathrm{C} / 24 \mathrm{~h}$. A suspensão de microrganismos leveduriformes foi preparada de modo similar. Após a padronização da turvação (equivalente ao tubo 0,5 da escala Mac Farland), foram preparadas diluições sucessivas de 1:50 e 1:20 em caldo RPMI 1640 tamponado; volumes de $100 \mu \mathrm{L}$ foram transferidos para as cavidades de uma microplaca estéril, contendo $100 \mu \mathrm{L}$ do caldo RPMI acrescido das diferentes concentrações intermediárias do extrato a ser testado. As placas foram incubadas a $35^{\circ} \mathrm{C} / 48 \mathrm{~h}$. As CIMs (concentrações inibitórias mínimas) foram determinadas com base na menor concentração do extrato que inibiu completamente o crescimento microbiano.

Para a preparação da solução estoque do extrato EtOH, este foi solubilizado em mistura de dimetilsulfóxido:polissorbato 80 a $0,5 \%$ (1:4), a fim de obter-se solução na concentração de $25 \mathrm{mg} / \mathrm{mL}$. Para a preparação da solução estoque do extrato $\mathrm{CH}_{2} \mathrm{Cl}_{2}$, o polissorbato $80 \mathrm{a}$ $0,5 \%$ foi substituído por polissorbato 80 a $10 \%$. As concentrações intermediárias dos extratos foram preparadas diluindo-se a solução estoque no meio apropriado, caldo Mueller-Hinton para bactérias e caldo RPMI 1640 para fungos. As concentrações finais testadas foram de 0,025 ; 0,$05 ; 0,25 ; 0,5 ; 1 ; 2,5$ e $5 \mathrm{mg} / \mathrm{mL}$. Os testes foram executados em triplicata.

Os microrganismos testados incluíram isolados clínicos e cultivos padrões de bactérias e fungos patogênicos, sendo estes listados na Tabela I.

\section{Isolamento}

$\mathrm{O}$ fracionamento preliminar do extrato $\mathrm{CH}_{2} \mathrm{Cl}_{2}$ $(11,7 \mathrm{~g})$ foi realizado por cromatografia em coluna (CC) flash sobre sílica-gel $(60 \times 4 \mathrm{~cm})$ utilizando diclorometano como fase móvel, sendo coletadas 17 frações de $250 \mathrm{~mL}$. As frações 2 e $3(4,68 \mathrm{~g})$ foram reunidas, concentradas e submetidas novamente à CC flash $(49,5 \times 4 \mathrm{~cm})$ utilizando hexano como fase móvel, sendo coletadas 27 frações de $100 \mathrm{~mL}$. As frações 2 a 11 (3,03 g) foram reunidas, concentradas e submetidas a fracionamento por $\mathrm{CC}(52,5 \times 4 \mathrm{~cm})$ utilizando sílica-gel impregnada com solução de nitrato de prata à 10\% (STAHL, 1969) e hexano:acetato de etila (98:2) como fase móvel, sendo coletadas 350 frações de $20 \mathrm{~mL}$. As frações 116 a 145 foram reunidas e evaporadas sob pressão reduzida, resultando em 271,3 mg do composto 1 .

Composto 1. Ressonância magnética nuclear de ${ }^{1} \mathrm{H}$ $\left(400 \mathrm{MHz}, \mathrm{CDCl}_{3}\right): \delta(\mathrm{ppm}) 0,82$ e $0,87(\mathrm{~d}, J 6,8 ; 6,72$ $\mathrm{Hz}, 12-\mathrm{H}$ e $13-\mathrm{H}), \sim 1,44(m, 11-\mathrm{H}), \sim 1,45(m, 8-\mathrm{H}), 1,52$ $(s, 14-\mathrm{H}), 2,00(m, 7-\mathrm{H}), \sim 2,00(m, 2-\mathrm{H}), 2,10$ ( $d d d, J \sim 5,0$; $\sim 7,8$; 12,8 Hz, 3-H), 2, 25 ( $m, 9-\mathrm{H}), \sim 2,35$ ( $m, 2-\mathrm{H}^{\prime}$ ), $\sim 2,43$ ( $\left.m, 3-\mathrm{H}^{\prime}\right), 4,75$ ( $\left.d, J 2,4 \mathrm{~Hz}, 15-\mathrm{H}\right), 4,80(d, J \sim 2,0$ $\left.\mathrm{Hz}, 15-\mathrm{H}^{\prime}\right), 5,14$ ( $d d, J 4,8$ e 11,6 Hz, 1-H), 5,26 (dd, $J 10$ e 16,0 Hz, 6-H), 5,79 (d, $J 15,6$ Hz, 5-H); Ressonância magnética nuclear de ${ }^{13} \mathrm{C}\left(100 \mathrm{MHz}, \mathrm{CDCl}_{3}\right): \delta(\mathrm{ppm}) 15,9$ (C-14), 19,3, 20,8 (C-12, C-13), 26,5 (C-8), 29,2 (C-2), 32,8 (C-11), 34, 5 (C-3), 40,7 (C-9), 52,9 (C-7), 109, 0 (C15), 129,6 (C-1), 133,5 (C-6), 134,0 (C-10), 135,5 (C-5), 148,8 (C-4); Espectrometria de massas (IE, $70 \mathrm{eV}$, TR = 
$30,7 \mathrm{~min}) \mathrm{m} / z(\%): 204(M+, 12), 161(100), 147(6)$, 133(21), 119(42), 105(66), 93(27), 91(66), 79(40), 77(38), 67(20), 55(22), 41(59).

\section{Procedimentos experimentais gerais}

Ressonância magnética nuclear de ${ }^{1} \mathrm{H}$ e de ${ }^{13} \mathrm{C}$ : BRUCKER DPX-400 ( ${ }^{1} \mathrm{H}: 400 \mathrm{MHz} ;{ }^{13} \mathrm{C}: 100 \mathrm{MHz}$, em $\mathrm{CDCl}_{3}$ ). CC: sílica-gel 60 , tamanho de partícula 63 a $200 \mathrm{~mm}$ (Merck). CCD: cromatofolhas de sílica-gel (Merck) $\mathrm{GF}_{254}$; detecção: anisaldeído- $\mathrm{H}_{2} \mathrm{SO}_{4}$. Cromatografia gasosa acoplada à espectrometria de massas: Sistema hifenado AGILENT 5973 equipado com um detector seletivo de massas 5973 e um injetor automático; parâmetros: split inlet 1:100; gás carreador: $\mathrm{He}(1 \mathrm{~mL} / \mathrm{min})$; coluna capilar de sílica fundida HP-5 MS (Hewlett Packard, fenilmetilpolisiloxano 5\%, $30 \mathrm{~m}$ de comprimento, $0,25 \mathrm{~mm}$ d.i., espessura do filme: $0,25 \mathrm{~mm}$ ); programa de análise: $40^{\circ} \mathrm{C}$ (Ti) por $4 \mathrm{~min}, 40-260^{\circ} \mathrm{C}, 4^{\circ} \mathrm{C} / \mathrm{min}$; temperatura do injetor: $220^{\circ} \mathrm{C}$; energia de ionização: $70 \mathrm{eV}$; banco de dados: NIST, 1998.

\section{RESULTADOS E DISCUSSÃO}

Os resultados da atividade antimicrobiana dos extratos $\mathrm{CH}_{2} \mathrm{Cl}_{2}$ e EtOH de $S$. heterotrichius encontram-se na Tabela I.

TABELA I - Concentrações inibitórias mínimas (CIM) obtidas para os extratos $\mathrm{CH}_{2} \mathrm{Cl}_{2}$ e EtOH de $S$. heterotrichius.

\begin{tabular}{lcc}
\hline \multirow{2}{*}{ Microrganismos } & \multicolumn{2}{c}{$\mathrm{CIM}(\mathrm{mg} / \mathrm{mL})$} \\
& \multicolumn{2}{c}{ dos extratos } \\
& $\mathrm{CH}_{2} \mathrm{Cl}_{2}$ & EtOH \\
\hline Candida albicans ATCC 44373 & $>5$ & $>5$ \\
C. dubliniensis BRCD 12 & $>5$ & $>5$ \\
C. lusitaniae EPM 50 & $>5$ & $>5$ \\
C. krusei FMUSP 1023 & 0,25 & $>5$ \\
C. parapsilosis HUSM 523 & $>5$ & $>5$ \\
Escherichia coli ATCC 25922 & $>5$ & $>5$ \\
Staphylococcus aureus ATCC 25923 & 2,5 & $>5$ \\
\hline
\end{tabular}

Os resultados da Tabela I indicam para o extrato $\mathrm{CH}_{2} \mathrm{Cl}_{2}$ de $\mathrm{S}$. heterotrichius moderada atividade antifúngica frente a Candida krusei (CIM de $0,25 \mathrm{mg} / \mathrm{mL}$ ). Cabe ressaltar que Candida krusei tem evidenciado problemático perfil de suscetibilidade a antifúngicos poliênicos e azólicos; sua resistência ao fluconazol é notória e estendese a triazólicos como o itraconazol (Sanglard, Odds, 2002).
A atividade antibacteriana frente a Staphylococcus aureus (CIM de 2,5 mg/mL) também deve ser considerada devido aos elevados percentuais de resistência que esta espécie apresenta diante de toda classe dos beta-lactâmicos. Os isolados de $S$. aureus resistentes à meticilina (MRSA) têm sido desafiadores e a constatação da resistência à vancomicina tem tornado o espectro de suscetibilidade de $S$. aureus crítico, contribuindo com elevadas taxas de mortalidade (Liu, Chambers, 2003). Neste contexto, a busca por novas moléculas que o inibam tanto in vivo como in vitro tem importância visando futuras aplicações, sejam clínicas ou mesmo na desinfecção de ambientes hospitalares. Portanto, estes valores de CIM, embora elevados, podem ser considerados relevantes devido às propriedades patogênicas destes microrganismos. Já o extrato EtOH não demonstrou atividade antimicrobiana nas concentrações testadas.

A atividade do extrato $\mathrm{CH}_{2} \mathrm{Cl}_{2}$ sobre um microrganismo Gram-positivo ( $S$. aureus) e ausência de atividade frente ao Gram-negativo testado (E. coli) estão de acordo com as informações contidas na literatura, que relatam maior sensibilidade dos primeiros frente aos metabólitos vegetais (Burt, 2004). Segundo Holley e Patel (2005), a membrana dual apresentada pelas bactérias Gram-negativas forma um envelope complexo, sendo responsável pela menor sensibilidade destes microrganismos frente aos extratos vegetais.

Atividade antimicrobiana já foi comprovada para várias espécies de Senecio, entre elas, Senecio maranguensis O. Hoffm. (Cos et al., 2002), S. desiderabilis Vellozo (Deuschle, 2006) e S. graveolens Wedd. (Pérez, Agnese, Cabrera, 1999).

Uma vez que a literatura descreve o gênero Senecio como sendo rico em terpenóides, compostos que freqüentemente apresentam atividade antimicrobiana, e o extrato $\mathrm{CH}_{2} \mathrm{Cl}_{2}$ mostrou-se ativo contra dois dos microrganismos testados, partiu-se para o seu fracionamento, visando isolar a(s) substância(s) ativa(s).

$\mathrm{O}$ composto 1 (Figura 1), isolado do extrato $\mathrm{CH}_{2} \mathrm{Cl}_{2}$ de $S$. heterotrichius foi identificado como sendo o germacreno D por comparação de seus dados espectroscópicos com os da literatura (Rostelien et al., 2000; Steliopoulos et al., 2002; Deuschle, 2007). Este sesquiterpeno já foi isolado de várias espécies de Senecio, mas até o momento sua presença não havia sido descrita em S. heterotrichius. A ocorrência freqüente do germacreno D no gênero Senecio pode ser explicada pelo fato deste composto ser um intermediário comum na biossíntese de outros hidrocarbonetos sesquiterpênicos, que por sua vez, dão origem a derivados de estruturas mais complexas (Büllow, König, 2000). O germacreno D é biossintetizado a partir do precursor difosfato de farnesila, através da catálise por 


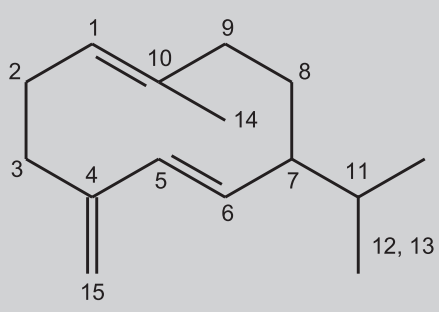

FIGURA 1 - Estrutura química do composto 1.

sesquiterpeno-sintases (ciclases). Assim como a maioria dos sesquiterpenóides existentes na natureza, o composto isolado é uma molécula quiral, existindo na forma de dois enantiômeros, $(+)$ e (-) (Koenig, 2001). Cada enantiômero do germacreno D é produzido por uma rota metabólica diferente, que requer diferentes enzimas (Huntington, Kinnel, 2006). Estudos visando a determinação e/ou quantificação do(s) enantiômero(s) do germacreno D isolado(s) de S. heterotrichius, não foram realizados. Para este composto é relatada atividade inibidora do apetite de insetos (Petrakis et al., 2005), tripanossomicida in vitro e ausência de citotoxicidade frente a Artemia salina (Biavatti et al., 2001). Frente a patógenos humanos, a atividade antimicrobiana do germacreno D já foi avaliada por dois métodos distintos: difusão em ágar (Biavatti et al., 2001) e microdiluição em caldo (Deuschle et al., no prelo). Em ambos os casos, o germacreno D mostrou-se inativo contra Bacillus subtilis, B. cereus, Staphylococcus aureus, Pseudomonas aeruginosa, Escherichia coli, Micrococcus roseus, M. luteus, Candida albicans, C. dubliniensis, $C$. krusei e Saccharomyces cerevisiae nas concentrações testadas (até $5 \mathrm{mg} / \mathrm{mL}$ ). Portanto, estes resultados indicam que o composto isolado não é o responsável pela atividade antimicrobiana do extrato $\mathrm{CH}_{2} \mathrm{Cl}_{2}$ detectada neste ensaio. No entanto, o germacreno D é um dos constituintes majoritários de óleos essenciais com atividade antimicrobiana comprovada, extraídos de diferentes espécies vegetais (Juteau et al., 2002; Gonzaga et al., 2003; Iacobellis et al., 2005; Chavan, Shinde, Nirmal, 2006) e pode estar contribuindo para a atividade antimicrobiana dos(s) constituinte(s) ativo(s) do extrato $\mathrm{CH}_{2} \mathrm{Cl}_{2}$ de $S$. heterotrichius. Segundo Cowan (1999), a lipofilia de hidrocarbonetos de estrutura terpênica permite a sua partição nos lipídeos da membrana celular, aumentando a permeabilidade desta. Esta propriedade pode facilitar a penetração de agentes antimicrobianos no interior celular e assim proporcionar aumento de atividade, constituindo-se numa explicação plausível para as interações positivas de constituintes de natureza sesquiterpenóide com antibióticos de uso conven- cional (Brehm-Stecher, Johnson, 2003; Ríos, Recio, 2005).

A atividade inibitória do extrato $\mathrm{CH}_{2} \mathrm{Cl}_{2}$ frente a Candida krusei e Staphylococcus aureus poderia ser de responsabilidade dos alcalóides pirrolizidínicos (APs), constituintes comuns no gênero Senecio, já descritos para S. heterotrichius (Krebs, Carl, Habermeh, 1996; Trigo et al., 2003). A atividade antimicrobiana já foi comprovada para esta classe de constituintes (Reina et al., 1995; Singh, Sahu, Singh, 2002), sendo conseqüência de sua capacidade em reagir com componentes nucleofílicos celulares como ácidos nucléicos (DNA) e proteínas, após sofrer desidrogenação catalisada por monooxigenases do sistema citocromo P450, e assim, interromper o ciclo celular do microrganismo (Prakash et al., 1999).

\section{ABSTRACT}

\section{Antimicrobial activity of Senecio heterotrichius DC.}

This work describes the evaluation of the antimicrobial activity of $\mathrm{CH}_{2} \mathrm{Cl}_{2}$ and EtOH extracts obtained by the maceration of the aerial parts of Senecio heterotrichius $D C$. against bacterial and fungal pathogens by broth microdilution method. The $\mathrm{CH}_{2} \mathrm{Cl}_{2}$ extract showed the best activity against Candida krusei (MIC $0.25 \mathrm{mg} / \mathrm{mL}$ ) and a moderate activity against Staphyllococcus aureus (MIC $2.5 \mathrm{mg} / \mathrm{mL}$ ). The EtOH extract was inactive against the tested microorganisms. Besides, one of the main constituents of $\mathrm{CH}_{2} \mathrm{Cl}_{2}$ extract was isolated, and its spectroscopic analysis indicated the presence of a sesquiterpene identified as germacrene D.

UNITERMS: Senecio heterotrichius DC/antimicrobial activity. Germacrene D. Asteraceae.

\section{AGRADECIMENTOS}

Ao botânico Marcos Sobral, pela identificação e auxílio na coleta do material vegetal. Apoio financeiro: CAPES e CNPq.

\section{REFERÊNCIAS BIBLIOGRÁFICAS}

ABDO, S.; DE BERNARDI, M.; MARINONI, G.; MELLERIO, G.; SAMANIEGO, S.; VIDARI, G.; FINZI, P.V. Furanoeremophilanes and other constituents from Senecio canescens. Phytochemistry, v.31, n.11, p.3937-3941, 1992. 
BIAVATTI, M.W.; VIEIRA, P.C.; DA SILVA, M.F.G.F.; FERNANDES， J.B.; ALBUQUERQUE， S.; MAGALHÃES, C.M.I.; PAGNOCCA, F.C. Chemistry and bioactivity of Raulinoa echinata Cowan, an endemic Brazilian Rutaceae species. Phytomedicine, v.8, n.2, p.121-124, 2001.

BREHM-STECHER, B.F.; JOHNSON, E.A. Sensitization of Staphylococcus aureus and Escherichia coli to antibiotics by the sesquiterpenoids nerolidol, farnesol, bisabolol, and apritone. Antimicrob. Agents Chemother., v.47, n.10, p.3357-3360, 2003.

BOHLMANN, F.; ZIESCHE, J. Sesquiterpenes from three Senecio species. Phytochemistry, v.20, n.3, p.469-472, 1981.

BÜLLOW, N.; KÖNIG, W.A. The role of germacrene D as a precursor in sesquiterpene biosynthesis: investigations of acid catalyzed, photochemically and thermally induced rearrangements. Phytochemistry, v.55, n.2, p.141-168, 2000.

BURT, S. Essential oils: their antibacterial properties and potential applications in foods - a review. Int. J. Food Microbiol., v.94, n.3, p.223-253, 2004.

CABRERA, A.L.; KLEIN, R.M. Compostas. In: REITZ, P.R. Flora ilustrada catarinense. Itajaí: Herbário Barbosa Rodrigues, 1975. p.136, 190-192.

CHAVAN, M.J.; SHINDE, D.B.; NIRMAL, S.A. Major volatile constituents of Annona squamosa L. bark. Nat. Prod. Res., v.20, n.8, p.754-757, 2006.

COS, P.; HERMANS, N.; DE BRUYNE, T.; APERS, S.; SINDAMBIWE, J.B.; VANDEN BERGHE, D.; PIETERS, L.; VLIETINCK, A.J. Further evaluation of Rwandan medicinal plant extracts for their antimicrobial and antiviral activities. J. Ethnopharmacol., v.79, n.2, p.155-163, 2002.

COWAN, M.M. Plant products as antimicrobial agents. Clin . Microbiol. Rev., v.12, n.4, p.564-582, 1999.

DEUSCHLE, R.A.N.; CAMARGO, T.; FRANCESCATO, L.N.;ALVES, S.H.; HEINZMANN, B.M. Antimicrobial activity of Senecio desiderabilis Vellozo (Asteraceae). Acta Farm. Bonaer., v.25, n.3, p.356-359, 2006.
DEUSCHLE, R.A.N.; CAMARGO, T.; ALVES, S.H.; MALLMANN, C.A.; HEINZMANN, B.M. Fracionamento do extrato diclorometânico de Senecio desiderabilis Vellozo e avaliação da atividade antimicrobiana. Rev. Bras. Farmacogn., v.17, n.2, p.7175, 2007.

DUPRÉ, S.; GRENZ, M.; JAKUPOVIC, J.; BOHLMANN, F.; NIEMEYER, H.M. Eremophilane, germacrane and shikimic acid derivatives from chilean Senecio species. Phytochemistry, v.30, n.4, p.1211-1220, 1991.

FORTIN, H.; VIGOR, C.; LOHÉZIC-LE DÉVÉHAT, F.; ROBIN, V.; LE BOSSÉ, B.; BOUSTIE, J.; AMOROS, M. In vitro antiviral activity of thirty-six plants from $\mathrm{La}$ Réunion Island. Fitoterapia, v.73, n.4, p.346-350, 2002.

GONZAGA, W.A.; WEBER, A.D.; GIACOMELLI, S.R.; SIMIONATTO, E.; DALCOL, I.I.; DESSOY, E.C.; MOREL, A.F. Composition and antibacterial activity of the essential oils from Zanthoxylum rhoifolium. Planta Med., v.69, n.8, p.773-775, 2003.

HOLLEY, R.A.; PATEL, D. Improvement in shelf-life and safety of perishable foods by plant essential oils and smoke antimicrobials. Food Microbiol., v.22, n.4, p.273-292, 2005.

HUNTINGTON, S.; KINNEL, R. Germacrene D: variation of enantiomers in Aster leaves and oviposition in the pearl crescent butterfly. Disponível em: <http:// www.chem.hamilton.edu/ rkinne $1 /$ summer_research_2004.html>.Acesso em: 09 nov. 2006.

IACOBELLIS, N.S.; LO CANTORE, P.; CAPASSO, F.; SENATORE, F. Antibacterial activity of Cuminum cyminum L. and Carum carvi L. essential oils. J. Agric. Food Chem., v.53, n.1, p.57-61, 2005.

JEFFREY, C.; HALLIDAY, P.; WILMOT-DEAR, M.; JONES, S.W. Generic and sectional limits in Senecio (Compositae): I. Progress report. Kew Bull., v.32, p.4767, 1977.

JUTEAU, F.; MASOTTI, V.; BESSIERE, J.M.; DHERBOMEZ, M.; VIANO, J. Antibacterial and antioxidant activities of Artemisia annua essential oil. Fitoterapia, v.73, n.6, p.532-535, 2002. 
KELMANSON, J.E.; JÄGER, A.K.; VAN STADEN, J. Zulu medicinal plants with antibacterial activity. $J$. Ethnopharmacol., v.69, n.3, p.241-246, 2000.

KOENIG, W.A. Enantioselective gas chromatography in stereochemical studies of natural compounds. Albany Molecular Research, Inc. - Technical Reports, v.6, n.52, 2001. p.2. (Apresentado no International Symposium, Exhibit \& Seminars on Chirality, 13, Orlando, 2001). Disponível em: <http://www.albmolecular.com/ chemlinks/reviews/vol06/no52/v06n52.pdf>. Acesso em: 09 nov. 2006.

KREBS, H.C.; CARL, T.; HABERMEHL, G.G. Pyrrolizidine alkaloid composition in six brazilian Senecio species. Phytochemistry, v.43. n.6, p.1227-1229, 1996.

LIU, C.; CHAMBERS, H.F. Staphylococcus aureus with heterogeneous resistance to vancomycin: epidemiology, clinical significance, and critical assessment of diagnostic methods. Antimicrob. Agents Chemother., v.47, n.10, p.3040-3045, 2003.

LIU, F.; NG, T.B. Antioxidative and free radical scavenging activities of selected medicinal herbs. Life Sci., v.66, n.8, p.725-735, 2000 .

LOIZZO, M.R.; TUNDIS, R.; STATTI, G.A.; MILJKOVICBRAKE, A.; MENICHINI, F.; HOUGHTON, P.J. Bioactive extracts from Senecio samnitum Huet. Nat. Prod. Res., v.20, n.3, p.265-269, 2006.

MANSOUR, R.M.A.; SALEH, N.A.M. Flavonoids of three local Senecio species. Phytochemistry, v.20, n.5, p.11801181, 1981.

NAVARRO GARCÍA, V.M.; GONZALEZ, A.; FUENTES, M.; AVILES, M.; RIOS, M.Y.; ZEPEDA, G.; ROJAS, M.G. Antifungal activities of nine traditional Mexican medicinal plants. J. Ethnopharmacol., v.87, n.1, p.85-88, 2003.

NCCLS. National Committee for Clinical Laboratory Standards. Performance standards for antimicrobial susceptibility testing. $12^{\text {th }}$ Informational Supplement, M100-S12. Wayne, 2002.

NCCLS. National Committee for Clinical Laboratory Standards. Reference method for broth dilution antifungical susceptibility testing of yeasts. 2.ed. Approved standard M27-A2. Wayne, 2002.
PASCUAL-VILLALOBOS, M.J.; ROBLEDO,A. Screening for anti-insect activity in Mediterranean plants. Ind. Crops Prod., v.8, n.3, p.183-194, 1998.

PÉREZ, C.; AGNESE, A.M.; CABRERA, J.L. The essential oil of Senecio graveolens (Compositae): chemical composition and antimicrobial activity tests. $J$. Ethnopharmacol., v.66, n.1, p.91-96, 1999.

PETRAKIS, P.V.; ROUSSIS, V.; PAPADIMITRIOU, D.; VAGIAS, C.; TSITSIMPIKOU, C. The effect of terpenoid extracts from 15 pine species on the feeding behavioural sequence of the late instars of the pine processionary caterpillar Thaumetopoea pityocampa. Behav. Processes, v.69, n.3, p.303-322, 2005.

PORTILLO, A.; VILA, R.; FREIXA, B.; ADZET, T.; CAÑIGUERAL, S. Antifungal activity of Paraguayan plants used in traditional medicine. J. Ethnopharmacol., v.76, n.1, p.93-98, 2001.

PRAKASH, A.S.; PEREIRA, T.N.; REILLY, P.E.B.; SEAWRIGHT, A.A. Pyrrolizidine alkaloids in human diet. Mutat. Res., Genet. Toxicol. Environ. Mutagen., v.443, n.1, p.53-67, 1999.

REINA, M.; MERICLI, A.H.; CABRERA, R.; GONZALEZ-COLOMA,A. Pyrrolizidine alkaloids from Heliotropium bovei. Phytochemistry, v.38, n.2, p.355$358,1995$.

RÍOS, J.L.; RECIO, M.C. Medicinal plants and antimicrobial activity. J. Ethnopharmacol., v.100, n.1/2, p.80-84, 2005.

ROSTELIEN, T.; BORG-KARLSON, A.-K.; FÄLDT, J.; JACOBSSON, U.; MUSTAPARTA, H. The plant sesquiterpene germacrene D specifically activates a major type of antennal receptor neuron of the tobacco budworm moth Heliothis virescens. Chem. Senses, v.25, n.2, p.141148, 2000.

SANGLARD, D.; ODDS, F.C. Resistance of Candida species to antifungal agents: molecular mechanisms and clinical consequences. Lancet, Infect. Dis., v.2, n.2, p.7385, 2002.

SINGH, B.; SAHU, P.M.; SINGH, S. Antimicrobial activity of pyrrolizidine alkaloids from Heliotropium subulatum. Fitoterapia, v.73, n.2, p.153-155, 2002. 
SMITH, L.W.; CULVENOR, C.C. Plant sources of hepatotoxic pyrrolizidine alkaloids. J. Nat. Prod., v.44, n.2, p.129-152, 1981.

STAHL, E. Thin-layer chromatography - a laboratory handbook. 2.ed. Berlin: Springer-Verlag, 1969. p.396402.

STELIOPOULOS, P.; WÜST, M.; ADAM, K.; MOSANDL, A. Biosynthesis of the sesquiterpene germacrene D in Solidago canadensis: ${ }^{13} \mathrm{C}$ and ${ }^{2} \mathrm{H}$ labeling studies. Phytochemistry, v.60, n.1, p.13-20, 2002.
TRIGO, J.R.; LEAL, I.R.; MATZENBACHER, N.I.; LEWINSOHN, T.M. Chemotaxonomic value of pyrrolizidine alkaloids in southern Brazil Senecio (Senecioneae: Asteraceae). Biochem. Syst. Ecol., v.31, n.9, p.1011-1022, 2003.

Recebido para publicação em 02 de janeiro de 2007. Aceito para publicação em 17 de maio de 2007. 